\title{
Research of Influence of Substitution of Renewable Energy Sources as a Part of the Scheme of Supply of Own Needs of Small and Micro-HPPS
}

\author{
Andrey A. Achitaev*a, \\ Aleksey A. Zhidkov ${ }^{b}$ and Nikolay S. Naumkin ${ }^{a}$ \\ ${ }^{a}$ Sayano-Shushensky Branch of Siberian Federal University \\ rp. Cheryomushki, Russian Federation \\ ${ }^{b}$ Novosibirsk State Technical University \\ Novosibirsk, Russian Federation
}

Received 03.06.2021, received in revised form 13.08.2021, accepted 01.09.2021

\begin{abstract}
One of the directions of modern development of renewable energy technologies in Russia is associated with the expansion of their application as part of the own needs of power plants. This article examines the study of the connection of renewable energy sources (RES) as part of the own needs of micro-hydroelectric power plants. The analysis of possibility of power supply of own needs of microHPP by wind power installation with use of the frequency Converter for the purposes of regulation of tension of own needs of station was carried out. The first part of the article is devoted to the analysis of publications on the current problem of the use of small and micro-hydroelectric power plants, as well as the description of the purpose and objectives of the study. The second section of the article presents mathematical models describing the connection of the wind turbine with a frequency Converter to work together with consumers of the station's own needs, for which the function of voltage regulation in the network is supposed to be performed. The law was formulated and an algorithm was constructed for the operation of the wind turbine with a load as part of the station's own needs. Note that this algorithm assumes continuous operation of the wind turbine without switching it according to the scheme of automatic reserve input, which leads to an increase in the installed capacity utilization factor. In the third part of the article the oscillograms of transient processes in the mode with and without wind turbines are considered. The short-circuit mode on the load and the influence of the wind turbine on the value of the recharge current are also considered. And in conclusion, the main conclusions on this work are formulated.
\end{abstract}

Keywords: autonomous electric power system, micro-HPP, own needs, voltage regulation, transients.

Citation: Achitaev A. A., Zhidkov A. A., Naumkin N. S. Research of influence of substitution of renewable energy sources as a part of the scheme of supply of own needs of small and micro-HPPS, J. Sib. Fed. Univ. Eng. \& Technol., 2021, 14(6), 656-666. DOI: $10.17516 / 1999-494 X-0341$

(C) Siberian Federal University. All rights reserved

This work is licensed under a Creative Commons Attribution-Non Commercial 4.0 International License (CC BY-NC 4.0).

* Corresponding author E-mail address: achitaevaa@gmail.com 


\title{
Исследование влияния замещения
}

\section{возобновляемых источников энергии \\ в составе схемы питания собственных нужд малых \\ и микро-ГЭС}

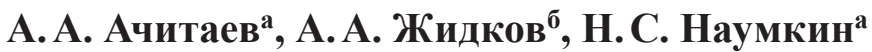 \\ ${ }^{a}$ Саяно-Шушенский филиал \\ Сибирского федерального университета \\ Российская Федерация, рп. Черемушки \\ ${ }^{6}$ Новосибирский государственный технический университет \\ Российская Федерация, Новосибирск
}

\begin{abstract}
Аннотация. Одно из актуальных направлений современного развития возобновляемой энергетики в России связано с применением их в составе собственных нужд электростанций. В данной статье рассматривается вопрос подключения возобновляемых источников энергии (ВИЭ) в составе собственных нужд микро-ГЭС. В исследовании был выполнен анализ возможности использования ветроэнергетической установки (ВЭУ) с преобразователем частоты для электроснабжения собственных нужд микро-ГЭС с учетом регулирования напряжения.

Первая часть статьи посвящена литературному обзору по проблематике использования малых и микро-ГЭС, а также описанию цели и задач исследования. Во втором разделе статьи представлены математические модели, описывающие подключение ВЭУ с преобразователем частоты на совместную работу с потребителями собственных нужд станции, для которых предполагается выполнение функции регулирования напряжения в сети. Был сформулирован закон и построен алгоритм для работы ВЭУ с нагрузкой в составе собственных нужд станции. Данный алгоритм предполагает непрерывную работу ВЭУ без ее переключения по схеме автоматического ввода резерва (АВР), что приводит к повышению коэффициента использования установленной мощности (КИУМ). В третьей части статьи описаны осциллограммы переходных процессов в режиме с учетом ВЭУ и без нее. Также рассмотрен режим короткого замыкания на нагрузке и влияние ВЭУ на величину тока подпитки. В заключение сформулированы основные результаты и выводы по данной работе.
\end{abstract}

Ключевые слова: автономная электроэнергетическая система, микро-ГЭС, собственные нужды, регулирование напряжения, переходные процессы.

Цитирование: Ачитаев, А. А. Исследование влияния замещения возобновляемых источников энергии в составе схемы питания собственных нужд малых и микро-ГЭС / А. А. Ачитаев, А. А. Жидков, Н. С. Наумкин // Журн. Сиб. федер. ун-та. Техника и технологии, 2021, 14(6). С. 656-666. DOI: 10.17516/1999-494X-0341

\section{Введение}

В настоящее время наблюдается рост использования ВИЭ в составе собственных нужд электростанции и подстанций. ПАО «РусГидро» приступает к реализации пилотного проекта по установке солнечных панелей на Нижне-Бурейской ГЭС. Установленная мощность новой солнечной электростанции (СЭС) равна 1275 кВт, ее ввод в эксплуатацию запланирован на конец 2019 г. По расчетам специалистов новый объект солнечной генерации будет вырабатывать 1,4 млн кВт•ч электроэнергии в год. Работа СЭС обеспечит снижение затрат электроэнергии на собственные нужды Нижне-Бурейской ГЭС, что позволит увеличить полезный отпуск электроэнергии, повысить эффективность работы ГЭС [1]. Данная статья посвящена формиро- 
ванию предложений по замещению потребления собственных нужд микро-ГЭС с применением ВИЭ на базе компонентов электронной генерации для решения задачи поддержания заданного значения напряжения в схемах собственных нужд. Рассмотрены разные режимы при анализе переходных процессов в целях параллельной работы генераторов и представлен анализ взаимного влияния работы ВЭУ в составе собственных нужд микро- и мини-ГЭС.

\section{Постановка задачи}

Объектом исследования стала автономная энергетическая система с микро-ГЭС установленной мощностью 8,1 кВт. Предусмотрено использование ВИЭ для обеспечения электроснабжения собственных нужд электростанции. Нагрузка энергосистемы составлена преимущественно из линейного активного сопротивления. В качестве объекта ВИЭ принята ВЭУ с магнитоэлектрическим генератором мощностью 1,1 кВт на 24 В, который снабжен собственным трансформатором на 1,7 кВА, преобразующим напряжение до 220 В. Подключение источников осуществляется через вставку постоянного тока, работающую по принципу автономного инвертора напряжения.

Схема электроснабжения представлена на рис. 1. В качестве местной нагрузки представлена резистивная тепловая нагрузка и ряд потребителей мощностью 7,6 кВт. Требуемая частота сети 50 Гц, напряжение сети 220 В. В составе собственных нужд учтена нагрузка. Схема выдачи мощности микро-ГЭС такова, что номинальное напряжение на выводе генератора совпадает с номинальным напряжением собственных нужд. Эта особенность приводит к формированию требований по раздельному регулированию напряжения (в схеме собственных нужд регулирование напряжения реализовано вставкой постоянного тока). Отдельно можно отметить, что повышенные требования по поддержанию напряжения в собственных нуждах связаны с использованием на данной микро-ГЭС уникальной технологии регулирования расхода воды. Наличие усовершенствованного органа регулирования требует поддержание постоянного уровня

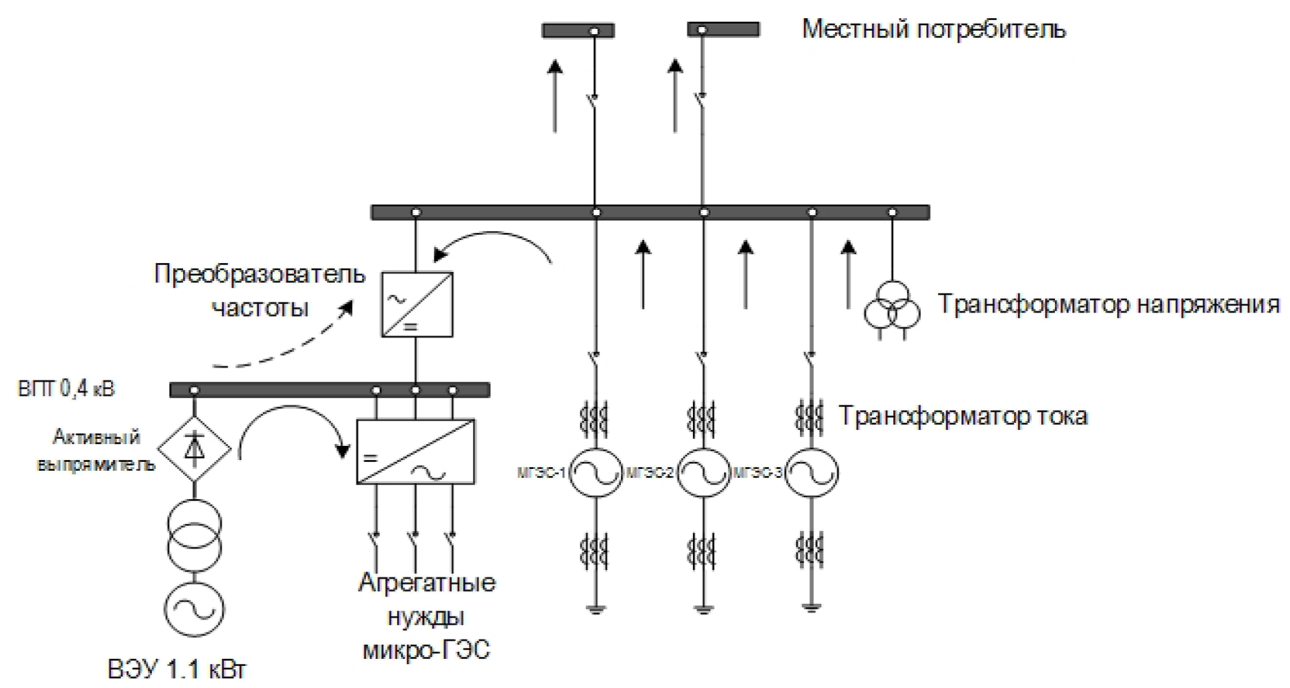

Рис. 1. Схема внешнего электроснабжения полигона

Fig. 1. External power supply diagram 
напряжения в сети. В настоящий момент в России не было задачи в составе малой ГЭС или микро-ГЭС решать проблему регулирования напряжения собственных нужд, которая является одной из самых важных задач для обеспечения надежности работы такой ГЭС и выдачи ее мощности в изолированную энергосистему.

В данной статье предлагается для поддержания напряжения использовать ВЭУ. При этом преобразователь частоты позволяет осуществить выдачу активной мощности в сеть благодаря двунаправленному многоуровневому преобразованию частоты. Таким образом, преобразователь позволяет выдавать излишки генерации ВЭУ в локальную электрическую сеть. Принцип регулирования напряжения собственных нужд - регулирование по реактивной мощности инвертора напряжения.

Вопросы стабилизации режимных параметров отражены в статье П. Лунду и Ю. Линдгрен [2]. Они занимались вопросами стабилизации режимных параметров электрических сетей путем использования активного управления тока для улучшения качества напряжения электрических сетей. Среди отечественных авторов можно выделить исследования О.В. Крюкова и А.В. Серебрякова [3], в которых были выделены основные алгоритмы стабилизации качества электрической энергии. Также можно отметить исследование С. А. Суякова, которое посвящено проблеме интеграции ВЭУ в сеть и формированию алгоритмов управления тока многоуровневого преобразователя частоты [4].

Цель данной статьи - анализ влияния источников с электронной генерацией на основе инверторов напряжения, установленных в собственных нуждах электростанции, на способность регулирования напряжения при различных режимах работы нагрузок, в том числе при коротком замыкании. Как известно, существует требование для собственных нужд, которое связано с поддержанием номинального напряжения. В настоящее время для решения данной проблемы используется вставка постоянного тока с функцией регулирования выдачи и потребления реактивной мощности через регулятор тока преобразователя частоты [5-12]. Однако использование инверторов на основе широтно-импульсной модуляции приводит к проблеме поддержания генераторного режима при пониженном напряжении в сети с последующим отключением источника в соответствии с характеристикой LVRT (англ. Low Voltage Ride Through), что вызывает потерю электроснабжения собственных нужд. Например, при близком коротком замыкании в связи со снижением напряжения происходит блокировка транзисторов, что влечет за собой прекращение выдачи активной мощности в сеть собственных нужд.

\section{Формирование математической модели и разработка законов управления напряжением}

Данная статья анализирует оценку влияния на качество напряжения во внешней сети, а также на режимные выходные параметры генераторов микро-ГЭС, элементов электронной генерации (автономный инвертор напряжения) в составе собственных нужд электростанций. Для этого составлена математическая модель, описывающая режимные параметры тока и напряжения во вращающейся системе координат.

Рассмотрим уравнение динамики вращения роторов электрических машин при их параллельной работе с источниками на основе ВИЭ с компонентами электронной генерации [13-15].

$$
-659-
$$




$$
\begin{aligned}
& {\left[\begin{array}{l}
V_{\alpha} \\
V_{\beta}
\end{array}\right]=\sqrt{\frac{2}{3}}\left[\begin{array}{ccc}
1 & -\frac{1}{2} & -\frac{1}{2} \\
0 & \frac{\sqrt{3}}{2} & -\frac{\sqrt{3}}{2}
\end{array}\right]\left[\begin{array}{l}
V_{a} \\
V_{b} \\
V_{c}
\end{array}\right]} \\
& {\left[\begin{array}{l}
i_{\alpha} \\
i_{\beta}
\end{array}\right]=\sqrt{\frac{2}{3}}\left[\begin{array}{ccc}
1 & -\frac{1}{2} & -\frac{1}{2} \\
0 & \frac{\sqrt{3}}{2} & -\frac{\sqrt{3}}{2}
\end{array}\right]\left[\begin{array}{l}
i_{a} \\
i_{b} \\
i_{c}
\end{array}\right],}
\end{aligned}
$$

где $i_{\alpha}, i_{\beta}-$ проекции тока по координатам $\alpha$ и $\beta$.

Данное уравнение вытекает из теории активной и реактивной мощности, позволяющей сформировать законы управления тока и напряжения по осям $d$ и $q$. Векторы напряжения и тока удобно представлять в плоскости вращения $\alpha, \beta$. Из формирования основного вектора напряжения и гармоник тока вытекает опорное напряжения, напряжение прямой последовательности:

$$
\left[\begin{array}{l}
V_{d} \\
V_{q}
\end{array}\right]=\left[\begin{array}{cc}
\cos (\theta) & \sin (\theta-3 \pi / 3) \\
-\sin (\theta) & \cos (\theta-3 \pi / 3)
\end{array}\right]\left[\begin{array}{c}
V_{\alpha} \\
V_{\beta}
\end{array}\right] .
$$

Из анализа приведенных выше уравнений следует, что для построения алгоритма управления необходимо принять следующую структуру, изображенную на рис. 2. Данный алгоритм

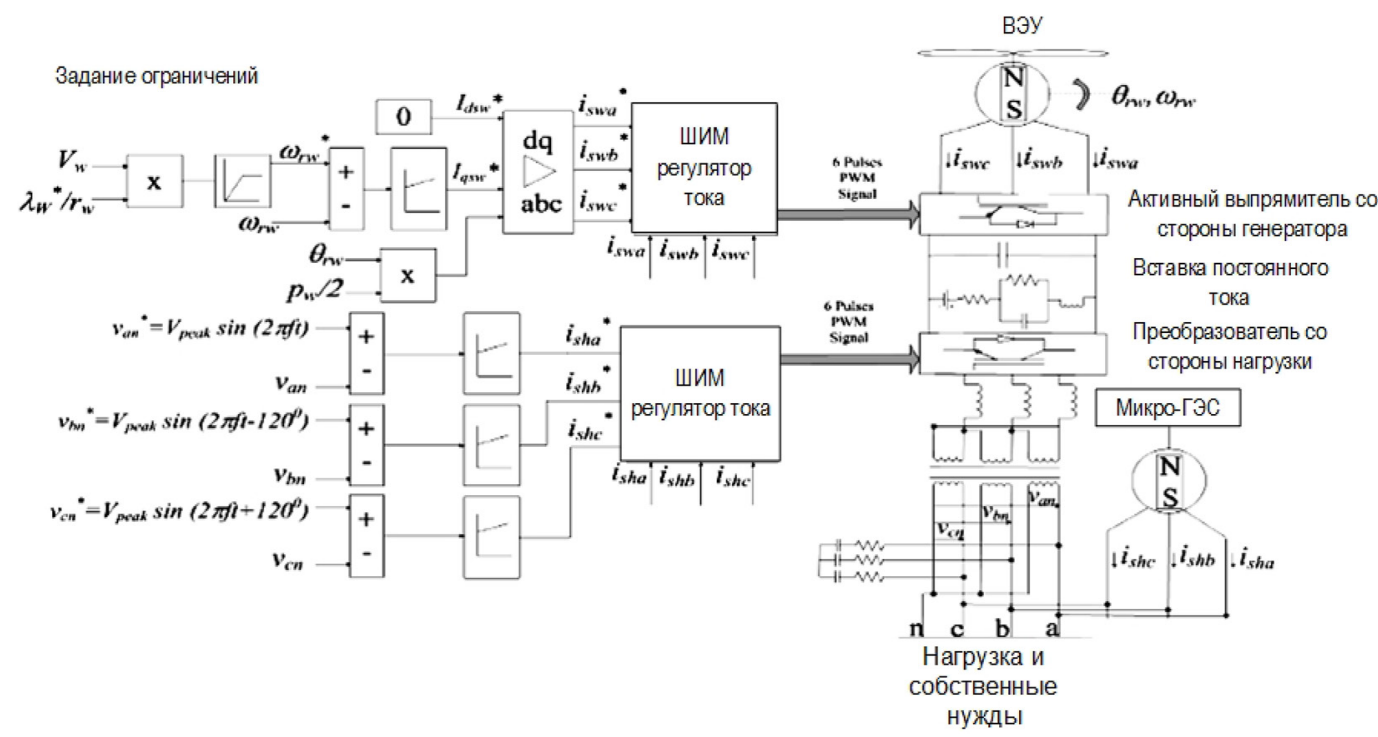

Рис. 2. Алгоритм управления регулятора тока преобразователя частоты ВЭУ

Fig. 2. Control algorithm of the current regulator of the wind turbine frequency converter

отражает возможность поддержки номинального напряжения в инверторе через регулирование соответствующего тока по оси $q$. Это способствует плавному регулированию заданного напряжения со стороны собственных нужд станции.

Функция, описывающая связь скорости вращения турбины ВЭУ со скоростью ветра, называется быстроходностью $\lambda$. Она выражается формулой

$$
-660-
$$




$$
\lambda=\frac{\omega R}{v_{0}}
$$

где $R$ - радиус турбины ВЭУ, м; $\omega$ - угловая скорость вращения турбины, рад/с; $v_{0}-$ скорость набегающего ветрового потока, м/с.

Для построения законов управления ПИ-регулятора тока ВЭУ по соответствующим осям примем следующий закон управления. Нужно отметить, что соответствующие токи по фазам раскладываются на составляющие по соответствующим видам источников электрической энергии [16]:

$$
\left\{\begin{array}{l}
i_{\mathrm{a}}(p)=i_{\text {sha }}(p-1)+K_{V}\left(\mathrm{v}_{\text {anerr }}(\mathrm{p})-\mathrm{v}_{\text {anerr }}(\mathrm{p}-1)\right)+\mathrm{K}_{\text {in }} v_{\text {anerr }}(p) \\
i_{\mathrm{b}}(p)=i_{\text {shb }}(p-1)+K_{V}\left(\mathrm{v}_{\text {bnerr }}(p)-\mathrm{v}_{\text {bnerr }}(\mathrm{p}-1)\right)+\mathrm{K}_{\text {in }} v_{\text {bnerr }}(p) \\
i_{\mathrm{c}}(p)=i_{\text {shc }}(p-1)+K_{V}\left(\mathrm{v}_{\text {cnerr }}(p)-\mathrm{v}_{\text {cnerr }}(\mathrm{p}-1)\right)+\mathrm{K}_{\text {in }} v_{\text {cnerr }}(p),
\end{array}\right.
$$

где $K_{v}$ - коэффициент усиления регулятора тока генератора ВЭУ; $K_{i n}-$ коэффициент усиления регулятора скорости турбины микро-ГЭС, $v_{\text {aerr, }} v_{b e r r}, v_{c e r r},-$ ошибки рассогласования векторов тока по фазам, $i_{s h a}, i_{s h b}, i_{s h c}-$ ограничение тока по фазам.

Проведя синтез законов регулирования, разработали алгоритм поддержания заданного напряжения в системе питания собственных нужд микро-ГЭС.

На рис. $3 a$ и 6 изображены графики логарифмической амплитудной частотной характеристики (ЛАЧХ) и откликов регуляторов тока со стороны преобразователя частоты ВЭУ и преобразователя микро-ГЭС. Пунктирная линия представляет регулятор тока преобразователя частоты ВЭУ, а сплошная - регулятор тока микро-ГЭС. Синтез данных регуляторов осуществлялся методом нулей и полюсов передаточной функции. Были составлены передаточные функции, отражающие влияние законов регулирования тока в замкнутой линейной системе. В табл. 1 представлены результаты сравнения передаточных функций замкнутой системы по току и полученной передаточной функции, отраженной в системе нелинейных уравнений (4). Из анализа переходных процессов можно увидеть разницу постоянных времени воздействия. Разница в постоянном времени по отклику на управление вызвана инерцией регулятора расхода воды и относительно быстрым воздействием на управление напряжения в контуре регулятора ветроэнергетической установки.

\section{Анализ результатов}

Для анализа переходных процессов было использовано программное обеспечение MatLab Simulink $^{\circledR}$ с применением метода расчета нелинейных дифференциальных уравнений по методу Дормана-Принца [16]. На рис. 4 изображены осциллограммы напряжения во внешней сети при подключении синхронных генераторов микро-ГЭС с постоянными магнитами на параллельную работу. Следует обратить внимание, что благодаря дополнительному регулированию напряжения собственных нужд за счет ВЭУ улучшилось качество напряжения во внешней сети за счет более стабильной работы регулятора расхода воды микро-ГЭС.

На рис. 5 отображены осциллограммы переходного процесса при подключении на параллельную работу генераторов микро-ГЭС. Из осциллограммы следует, что при подключении ВЭУ с использованием преобразователя частоты с функцией регулирования реактивной мощности наблюдается ровный график скорости вращения генератора. Колебания скорости вращения генераторов микро-ГЭС наблюдаются без применения регулирования тока ВЭУ и связаны с регули-

$$
-661-
$$




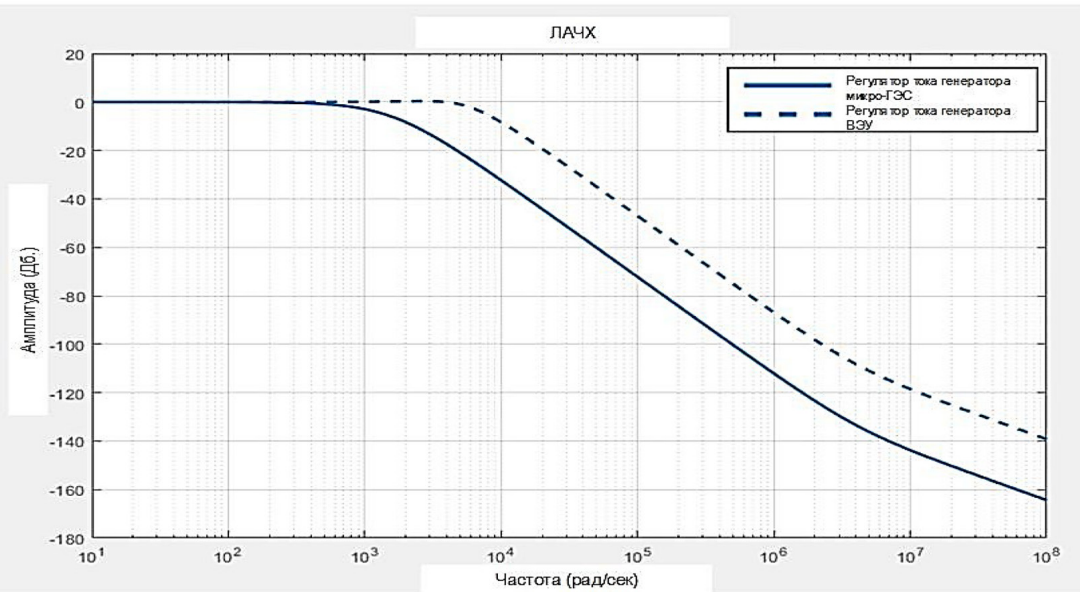

(a)

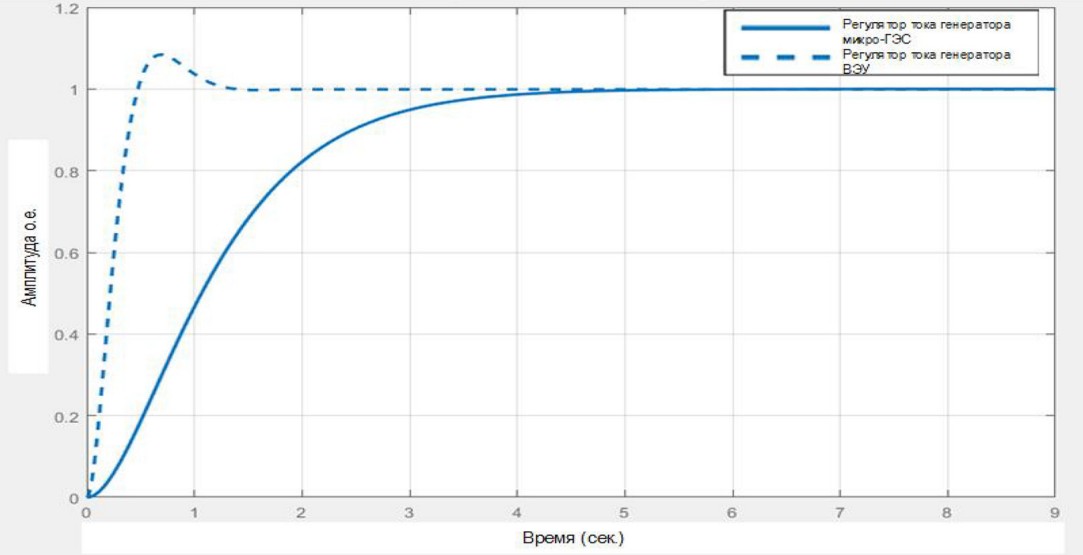

(б)

Рис. 3. Осциллограммы показателей качества переходных процессов: а - ЛАЧХ передаточной функции регулятора тока преобразователя со стороны ВЭУ; б - отклика по управляющему воздействию регулятора тока со стороны преобразователя генератора микро-ГЭС

Fig. 3. Oscillograms of transient processes: a - the BMP of the transfer function of the converter current controller from the WT side; $b$ - response to the control action of the current controller from the side of the micro hydro generator converter

Таблица 1. Характеристики замкнутых систем по контуру регулирования тока

Table 1. Characteristics of the automatic control system for current regulation

\begin{tabular}{|c|c|c|}
\hline Параметр & $\begin{array}{c}\text { Замкнутая система } \\
\text { по регулированию тока } \\
\text { преобразователя частоты } \\
\text { со стороны микро-ГЭС }\end{array}$ & $\begin{array}{c}\text { Замкнутая система } \\
\text { по регулированию току } \\
\text { преобразователя частоты } \\
\text { со стороны ВЭУ }\end{array}$ \\
\hline П & $-1,113$ & $-0,668$ \\
\hline И & -7022 & -4453 \\
\hline Перерегулирование & $3.91 \%$ & $8.4 \%$ \\
\hline Коэффициент затухания & 60.8 дБ & 57.2 дБ \\
\hline
\end{tabular}




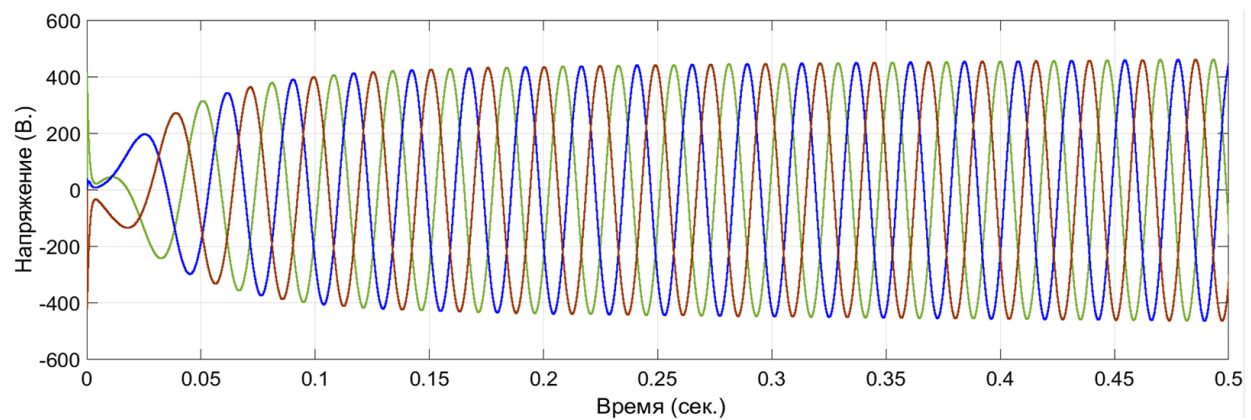

(a)

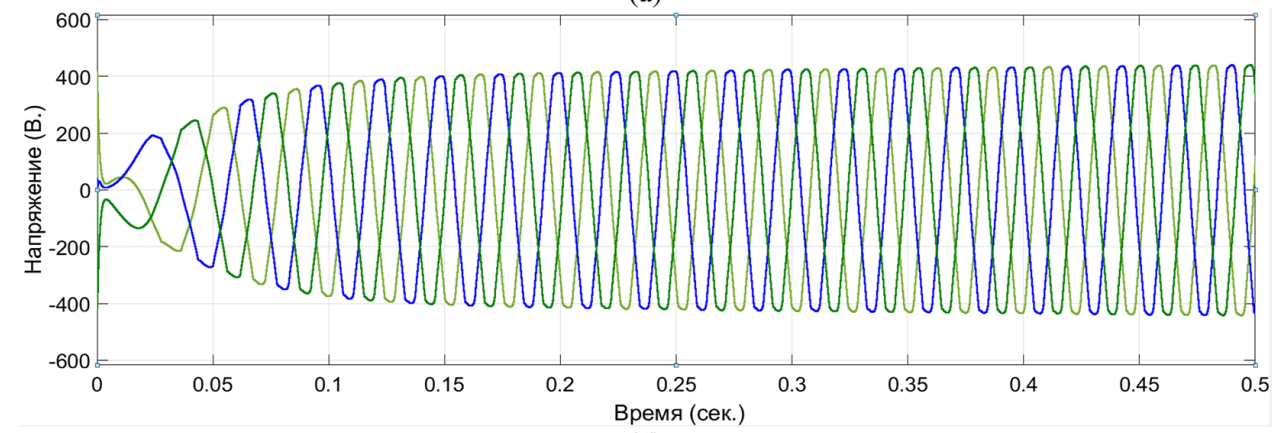

(б)

Рис. 4. Осциллограммы выходного напряжения агрегатов микро-ГЭС: а - учитывающие регулирование напряжения собственных нужд за счет преобразователя частоты ВЭУ; б - не учитывающие использования регулятора преобразователя со стороны ВЭУ

Fig. 4. Oscillograms of the output voltage of micro hydro generators: a - with the auxiliary voltage regulation by the wind turbine frequency converter; $b$ - without the use of the wind turbine frequency converter

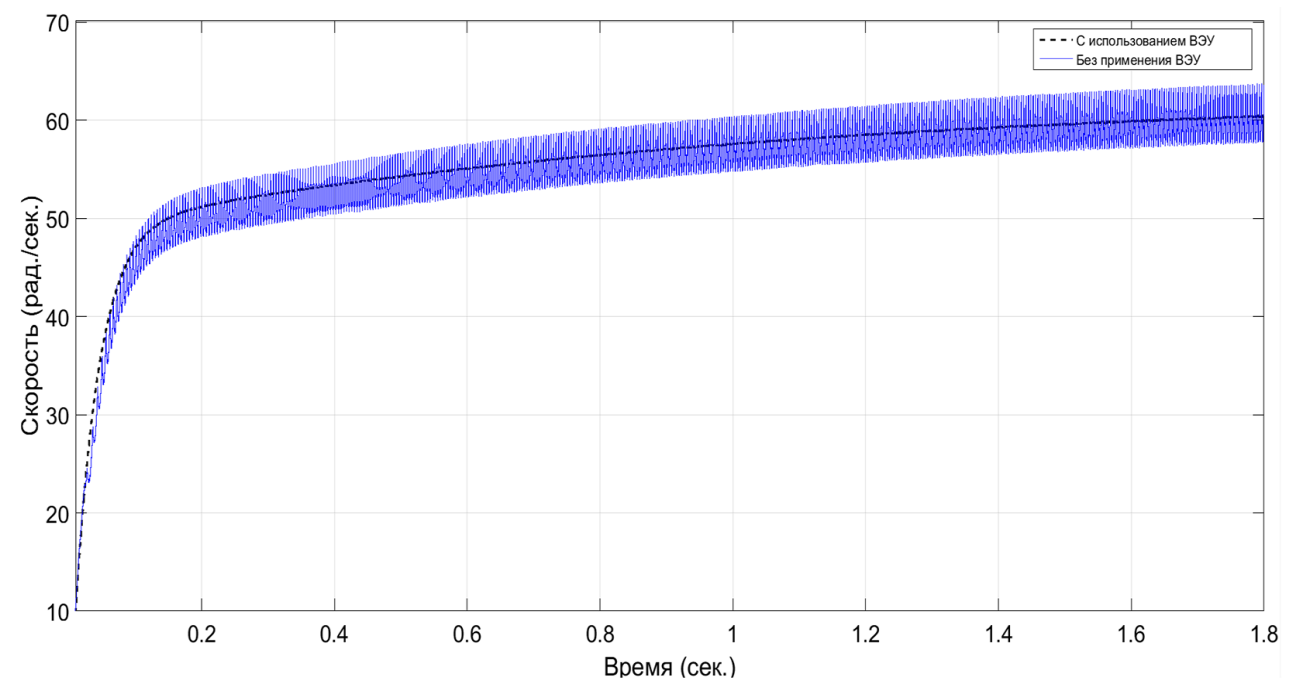

Рис. 5. Осциллограмма переходного процесса при подключении многополюсных синхронных генераторов на параллельную работу

Fig. 5. Waveform of the transient process when connecting multi-pole synchronous generators to parallel operation 
рованием тока только со стороны инвертора собственных нужд микро-ГЭС, чего недостаточно для стабилизации напряжения. Это вызывает колебания частоты вращения генераторов.

Рассмотрим влияние регулирования напряжения собственных нужд за счет ВЭУ в режиме короткого замыкания на местной нагрузке. На рис. 6 представлены осциллограммы напряжений в сети при коротком замыкании. На них можно увидеть снижение напряжения в обоих слу-

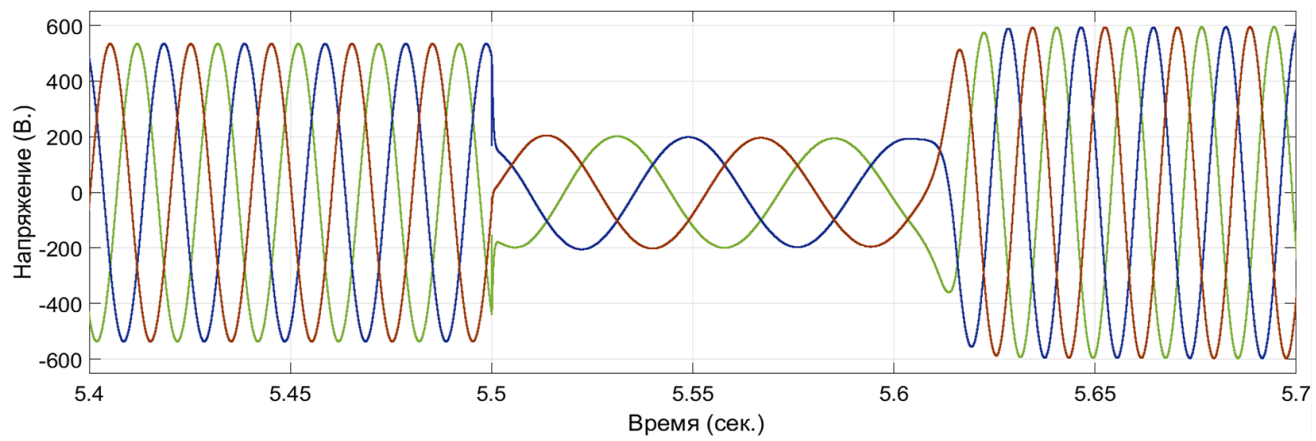

(a)

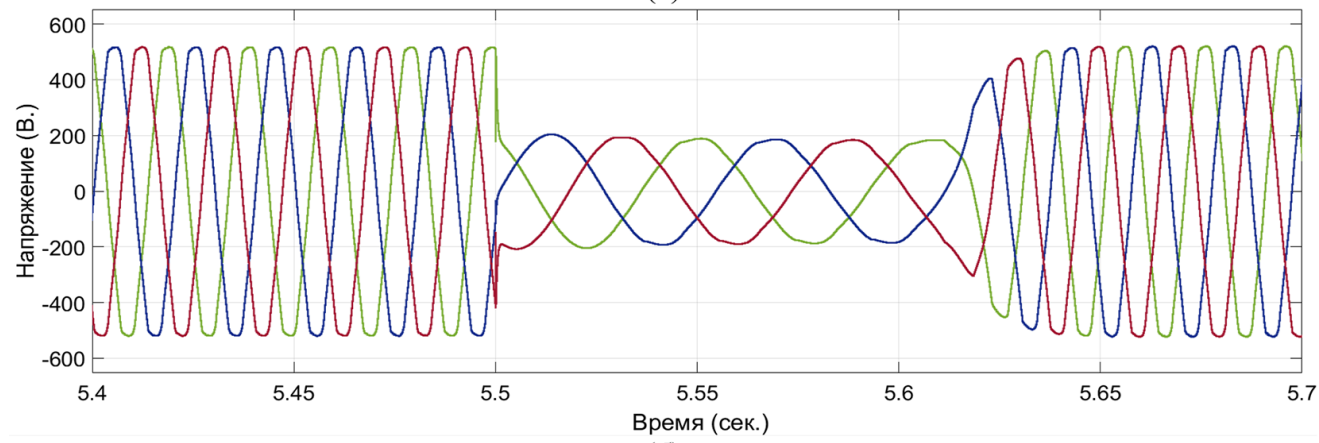

(б)

Рис. 6. Осциллограмма напряжений при коротком замыкании: a - с учетом использования ВЭУ; б - без учета использования ВЭУ

Fig. 6. Oscillogram of voltages at short circuit: $a$ - with the use of the wind turbine; $b$ - without the use of the wind turbine

чаях почти в два раза. Период короткого замыкания составляет 100 мс. Анализ осциллограмм выявляет, что использование в составе собственных нужд ВЭУ и преобразователя частоты с функцией регулирования напряжения в режиме внешнего короткого замыкания позволяет повысить качество напряжения в сети. Кроме того, использование ВЭУ также дает возможность обеспечить резерв электроснабжения собственных нужд при отключении сетевого преобразователя частоты в соответствии с характеристикой LVRT.

\section{Выводы}

На основании проведенного исследования были проанализированы два варианта электроснабжения собственных нужд микро-ГЭС. После анализа режимов можно сказать о возможности использования ветроэнергетических установок с регулятором реактивной мощности для обеспечения электроснабжения собственных нужд мини- и микро-ГЭС, работающих 
в автономной энергосистеме. Из анализа также следует, что регулирование напряжения путем применения вставки постоянного тока с управлением по току по оси q не в полной мере обеспечивает потребность по поддержанию заданного уровня напряжения, так как в этом случае наблюдаются колебания скорости вращения генераторов микро-ГЭС. Применение средств электронной генерации со стороны ветроэнергетической установки позволяет поддержать номинальное значение напряжения в сети собственных нужд и в итоге за счет более стабильной работы регулятора расхода воды и других потребителей собственных нужд повлиять на качество выходных параметров электростанции. Приведенные осциллограммы переходных процессов в режиме короткого замыкания подтверждают работоспособность предлагаемого варианта электроснабжения собственных нужд при коротких замыканиях в электрической сети. Дальнейшее исследование связано с применением солнечной станции совместно с ветроэнергетической установкой и анализа электромеханической совместимости при их совместной работе при различных возмущающих воздействиях (резкие сбросы нагрузки и близкие короткие замыкания).

\section{Благодарности / Acknowledgements}

Исследование выполнено при финансовой поддержке РФФИ в рамках научного проекта № 19-38-90034.

The reported study was supported by RFBR, research project No. 19-38-90034.

\section{Список литературы / References}

[1] Солнечные панели мощностью 1275 кВт будут установлены на Нижне-Бурейской ГЭСрежим доступа: http://www.rushydro.ru/press/news/107567.html [дата обращения: 13.12.2019]. [Solar panels with a capacity of $1,275 \mathrm{~kW}$ will be installed at the Nizhne-Bureyskaya HPP-access mode: http://www.rushydro.ru/press/news/107567.html [accessed: 13.12.2019] (in Russian)]

[2] Lund P.D. et al. Review of energy system flexibility measures to enable high levels of variable renewable electricity. Renewable and Sustainable Energy Reviews, 2015, 45, 785-807.

[3] Крюков О.В., Серебряков А.В. Алгоритмы энергоэффективного управления ветроэнергетическими установками. Электричество. 2017, 6, 31-35. [Kryukov O.V., Serebryakov A. V. Algorithms for energy-efficient management of wind power plants. Electricity, 2017, 6, 31-35 (in Russian)]

[4] Суяков С.А. Проблемы интеграции ветроустановок в единую энергетическую систему России. Инженерный вестник Дона. 2014, 30(2). [Suyakov S. A. Problems of integration of wind turbines in the unified energy system of Russia. Engineering Bulletin of the Don, 2014, 30(2) (in Russian)]

[5] Ling Y. The fault ride through technologies for doubly fed induction generator wind turbines, Wind Engineering, 2016, 40(1), 31-49.

[6] Li, J., Yang, Q., Yao, P., Sun, Q., Zhang, Z., Zhang, M., \& Yuan, W. A Novel use of the Hybrid Energy Storage System for Primary Frequency Control in a Microgrid. Energy Procedia, 2016, 103, $82-87$.

[7] R. Cardenas, R. Pena, S. Alepuz, and G. Asher, Overview of control systems for the operation of DFIGs in wind energy applications, IEEE Trans. Ind. Electron., Jul. 2013 60(7), 2776-2798.

$$
-665-
$$


[8] J. Morren and S.W.H. de Haan, Ride through of wind turbines with doublyfed induction generator during a voltage dip, IEEE Trans. Energy Convers., Jun. 2005, 20(2), 435-441.

[9] C. Wessels, F. Gebhart, and R.W. Fuchs, Fault ride-through of a DFIG wind turbine using a dynamic voltage restorer during symmetrical and asymmetrical grid faults, IEEE Trans. Power Electron., Mar. 2011, 26(3), 807-815.

[10]A.D. Hansen and G. Michalke, Fault ride-through capability of DFIG wind turbines, Renew. Energy, 2007, 32(9), 1594-1610.

[11] G. Pannell, D J. Atkinson, and B. Zahawi, Minimum-threshold crowbar for a fault-ide-through grid-code-compliant DFIG wind turbine, IEEE Trans. Energy Convers., Sep. 2010, 25(3), 750-759.

[12]Huang $\mathrm{H}$. et al. Electronic power transformer control strategy in wind energy conversion systems for low voltage ride-through capability enhancement of directly driven wind turbines with permanent magnet synchronous generators (D-PMSGs), Energies, 2014, 7(11), 7330-7347.

[13]Chandran V.P., Murshid S., Singh B. Voltage and Frequency Regulation with Load Leveling of a PMSG Based Pico-Hydro System Using SLMS Control Algorithm, 2018 5th IEEE Uttar Pradesh Section International Conference on Electrical, Electronics and Computer Engineering (UPCON). IEEE, 2018, 1-6.

[14]Goel P.K. et al. Autonomous hybrid system using SCIG for hydropower generation and variable speed PMSG for wind power generation. 2009 International Conference on Power Electronics and Drive Systems (PEDS). IEEE, 2009, 55-60.

[15]Goel P.K. et al. Autonomous hybrid system using PMSGs for hydro and wind power generation. 2009 35th Annual Conference of IEEE Industrial Electronics. IEEE, 2009, 255-260.

[16]Lamichhane S., Mithulananthan N. Influence of wind energy integration on low frequency oscillatory instability of power system. 2014 Australasian Universities Power Engineering Conference (AUPEC). IEEE, 2014, 1-5. 